\title{
Existence domains for invariant reactions in binary regular solution phase diagrams exhibiting two phases
}

\author{
B NAGESWARA SARMA, S SRINIVAS PRASAD, S VIJAYVERGIYA, \\ V BHARATH KUMAR and S LELE*
}

Department of Metallurgical Engineering, Institute of Technology, Banaras Hindu University, Varanasi 221005 , India

MS received 25 February 2003

\begin{abstract}
The thermodynamic origin of various types of phase diagrams in simple binary systems exhibiting two phases (e.g. a liquid and a solid phase) has been examined using the regular solution model. The necessary conditions for the occurrence of each of these types are identified in terms of the appropriate intersections of the miscibility gap boundaries (in solid/liquid phases) and the liquidus/solidus/iso- $G$ curves. Thus, the regions of occurrence of the different types of possible phase diagrams in the space of the regular solution interchange energy parameters $\left(W^{\alpha}, W^{\beta}\right)$ are clearly delineated. This analysis makes it easier to make intelligent initial selections of model (energy) parameters for their optimization in the calculation of phase diagrams using thermodynamic models such as CALPHAD/CVM.
\end{abstract}

Keywords. Binary phase diagrams; invariant reactions; regular solution model.

\section{Introduction}

Using the regular solution model, van Laar (1908) has shown that many of the observed types of simple phase diagrams can be obtained by a systematic variation of the regular solution interchange energy parameters $\left(W^{\beta}\right.$ and $W^{\alpha}$ for liquid and solid phases, respectively) in the positive domain with $W^{\beta} \leq W^{\alpha}$. By varying $W^{\alpha}$ from very large positive values to zero (keeping $W^{\beta}=0$ ), he has obtained eutectic, peritectic + congruent minimum, isomorphous with a congruent minimum followed by simple isomorphous (without an extremum) phase diagrams. Except for the limiting case $\left(W^{\beta}=W^{\alpha}=0\right)$ of a simple isomorphous phase diagram, the last two types of phase diagrams display a miscibility gap in the solid phase at low temperatures. Further, an increase in $W^{\beta}$ from zero to $W^{\alpha}$ for large positive values of $W^{\alpha}$, resulted in the formation of eutectic, monotectic + eutectic followed by monotectic + peritectic phase diagrams, respectively. The thermodynamic conditions for the transitions among these types of phase diagrams have been clearly identified for the first time by him. Pelton and Thompson (1975), Oonk (1981), DeHoff (1993) and Chang and Chen (1997), among others, have shown the occurrence of several types of simple binary phase diagrams for different combinations of regular solution parameters.

While the pioneering analysis of van Laar is restricted to a positive octant of $\left(W^{\alpha}, W^{\beta}\right)$ space, none of the earlier

*Author for correspondence investigators have attempted to delineate the regions corresponding to all the types of possible regular solution binary phase diagrams. This is carried out in the present investigation.

\section{Thermodynamic background}

Let us consider two phases $\alpha$ and $\beta$ which exhibit regular solution behaviour. The molar free energy of say $\alpha$ phase can be expressed as

$$
\begin{aligned}
G^{\alpha}= & \left(1-x^{\alpha}\right){ }^{o} G_{\mathrm{A}}^{\alpha}+x^{\alpha}{ }^{o} G_{\mathrm{B}}^{\alpha}+\left(1-x^{\alpha}\right) x^{\alpha} W^{\alpha}+R T \\
& \left\{\left(1-x^{\alpha}\right) \ln \left(1-x^{\alpha}\right)+x^{\alpha} \ln \left(x^{\alpha}\right)\right\}
\end{aligned}
$$

where $x^{\alpha}$ is the mole fraction of $\mathrm{B}, W^{\alpha}$ the interchange energy parameter while ${ }^{o} G_{\mathrm{A}}^{\alpha}$ and ${ }^{o} G_{\mathrm{B}}^{\alpha}$ are the standard molar free energies of the pure components in the $\alpha$ phase. A similar expression can be written for the $\beta$ phase. The chemical potentials of the components A and $\mathrm{B}$ in $\alpha$ phase can be obtained from standard thermodynamics and are given by

$$
\begin{aligned}
& \mu_{\mathrm{A}}^{\alpha}={ }^{o} G_{\mathrm{A}}^{\alpha}+\left(x^{\alpha}\right)^{2} W^{\alpha}+R T \ln \left(1-x^{\alpha}\right), \\
& \mu_{\mathrm{B}}^{\alpha}={ }^{o} G_{\mathrm{B}}^{\alpha}+\left(1-x^{\alpha}\right)^{2} W^{\alpha}+R T \ln \left(x^{\alpha}\right) .
\end{aligned}
$$

From the conditions of equilibrium between $\alpha$ and $\beta$ phases that the chemical potentials of each component in both the phases be the same, we obtain, after simplification (van Laar 1908), 


$$
\begin{gathered}
T=\frac{\Delta H_{\mathrm{A}}+\left(x^{\beta}\right)^{2} W^{\beta}-\left(x^{\alpha}\right)^{2} W^{\alpha}}{\Delta S_{\mathrm{A}}-R \ln \left(\frac{1-x^{\beta}}{1-x^{\alpha}}\right)}, \\
T=\frac{\Delta H_{\mathrm{B}}+\left(1-x^{\beta}\right)^{2} W^{\beta}-\left(1-x^{\alpha}\right)^{2} W^{\alpha}}{\Delta S_{\mathrm{B}}-R \ln \left(\frac{x^{\beta}}{x^{\alpha}}\right)},
\end{gathered}
$$

where the free energies for the transformation from $\alpha$ to $\beta$ have been expressed in terms of the enthalpies $(\Delta H)$ and entropies $(\Delta S)$ of transformation of the pure components as in the following

$$
\Delta G_{\mathrm{A}}={ }^{o} G_{\mathrm{A}}^{\beta}-{ }^{o} G_{\mathrm{A}}^{\alpha}=\Delta H_{\mathrm{A}}-T \Delta S_{\mathrm{A}}=\left(T_{\mathrm{A}}-T\right) \Delta S_{\mathrm{A}},
$$

in which, $T_{\mathrm{A}}$ is the transformation temperature of pure A.

The slopes of the phase boundaries are given by the Van der Waals equations (Oonk 1981), according to which the slope of the solidus is given by

$$
\frac{\mathrm{d} T_{\text {sol }}}{\mathrm{d} x^{\alpha}}=\frac{d^{2} G^{\alpha} / \mathrm{d} x^{\alpha^{2}}}{\left(\mathrm{~d} S^{\alpha} / \mathrm{d} x^{\alpha}\right)-\left(S^{\beta}-S^{\alpha}\right) /\left(x^{\beta}-x^{\alpha}\right)},
$$

where $S^{\alpha}$ and $S^{\beta}$ are the entropies of the two phases at their respective equilibrium compositions, $x^{\alpha}$ and $x^{\beta}$. For the regular solution model being utilized, the numerator and the denominator are respectively given by

$$
\begin{gathered}
\frac{\mathrm{d}^{2} G^{\alpha}}{\mathrm{d} x^{\alpha^{2}}}=-2 W^{\alpha}+\frac{R T}{x^{\alpha}\left(1-x^{\alpha}\right)}, \\
\frac{\mathrm{d} S^{\alpha}}{\mathrm{d} x^{\alpha}}-\frac{S^{\beta}-S^{\alpha}}{x^{\beta}-x^{\alpha}}=\frac{R}{\left(x^{\beta}-x^{\alpha}\right)}\left[-\frac{x^{\beta} \Delta S_{\mathrm{B}}+\left(1-x^{\beta}\right) \Delta S_{\mathrm{A}}}{R}\right. \\
\left.+\left(1-x^{\beta}\right) \ln \left(\frac{1-x^{\beta}}{1-x^{\alpha}}\right)+x^{\beta} \ln \left(\frac{x^{\beta}}{x^{\alpha}}\right)\right] .
\end{gathered}
$$

The iso- $G$ curve (Oonk 1981) is the locus of points ( $x$, $T$ ) for which the free energies of two phases $\alpha$ and $\beta$ are equal, i.e.

$$
G^{\alpha}\left(x^{\alpha}=x, T_{0}\right)=G^{\beta}\left(x^{\beta}=x, T_{0}\right) .
$$

Substituting for $G^{\alpha}$ and $G^{\beta}$ from equations of the type (1) and simplifying, we obtain

$$
(1-x) \Delta G_{\mathrm{A}}+x \Delta G_{\mathrm{B}}+x(1-x) \Delta W=0,
$$

where $\Delta W=W^{\beta}-W^{\alpha}$. Substituting from (6) and an analogous one for component B in (11) and solving for $T_{0}$, we obtain

$$
T_{0}=\frac{(1-x) T_{\mathrm{A}} \Delta S_{\mathrm{A}}+x T_{\mathrm{B}} \Delta S_{\mathrm{B}}+x(1-x) \Delta W}{(1-x) \Delta S_{\mathrm{A}}+x \Delta S_{\mathrm{B}}} .
$$

For the special case when $\Delta S_{\mathrm{A}}=\Delta S_{\mathrm{B}}=\Delta S$, this relation simplifies to

$$
T_{0}=(1-x) T_{\mathrm{A}}+x T_{\mathrm{B}}+x(1-x) \frac{\Delta W}{\Delta S} .
$$

We shall recapitulate the relation (Oonk 1981) for a miscibility gap boundary in the $\alpha$ phase

$$
T_{\mathrm{mg}}^{\alpha}=\frac{\left(1-2 x^{\alpha}\right)}{R \ln \left[\left\{1-x^{\alpha}\right\} / x^{\alpha}\right]} W^{\alpha} .
$$

The maximum temperature corresponding to the miscibility gap boundary (the so-called consolute point) is given by

$$
T_{\mathrm{C}}^{\alpha}=\frac{W^{\alpha}}{2 R} .
$$

\section{Isomorphous systems}

While the subsequent arguments are valid for phase diagrams exhibiting any two phases $\alpha$ and $\beta$, we shall present the discussion treating $\alpha$ as the solid phase and $\beta$ as the liquid phase. We shall first consider isomorphous systems and obtain conditions for the presence or absence of a congruent extremum (maximum or minimum) in such systems. Since the condition for iso- $G$ curve $\left(G^{\beta}-\right.$ $G^{\alpha}=0$ ) happens to be one of the equilibrium conditions corresponding to the congruent extremum $\left(G^{\beta}-G^{\alpha}=0\right.$ and $\left.\mathrm{d} G^{\beta} / \mathrm{d} x-\mathrm{d} G^{\beta} / \mathrm{d} x=0\right)$, the congruent extremum in the iso- $G$ curve coincides with that in the $\alpha-\beta$ equilibrium boundaries. Hence the necessary condition for the occurrence of a congruent extremum can be found by differentiating $T_{0}$ with respect to $x$ and setting the derivative to zero.

$$
\frac{\mathrm{d} T_{0}}{\mathrm{~d} x}=\frac{\Delta T \Delta S_{\mathrm{A}} \Delta S_{\mathrm{B}}+\left[(1-x)^{2} \Delta S_{\mathrm{A}}-x^{2} \Delta S_{\mathrm{B}}\right] \Delta W}{\left[(1-x) \Delta S_{\mathrm{A}}+x \Delta S_{\mathrm{B}}\right]^{2}}=0,
$$

where $\Delta T=T_{\mathrm{B}}-T_{\mathrm{A}}$. Thus, the composition for the maximum or minimum is given by

$$
x^{m}=\frac{-\Delta S_{\mathrm{A}} \Delta W \pm \sqrt{\Delta S_{\mathrm{A}} \Delta S_{\mathrm{B}}} \sqrt{\Delta W^{2}+\Delta T\left(\Delta S_{\mathrm{B}}-\Delta S_{\mathrm{A}}\right) \Delta W}}{\left(\Delta S_{\mathrm{B}}-\Delta S_{\mathrm{A}}\right) \Delta W},
$$

in which the positive (negative) sign is applicable for positive (negative) values of $\Delta W$.

A considerable simplification occurs for the case of $\Delta S_{\mathrm{A}}=\Delta S_{\mathrm{B}}=\Delta S$.

$$
x^{m}=\frac{1}{2}+\frac{\Delta T \Delta S}{\Delta W} \text {. }
$$


On substitution of this value in (13), the temperature for the congruent extremum for this special case is given by

$$
T_{0}^{m}=\frac{1}{2}\left(T_{\mathrm{B}}+T_{\mathrm{A}}\right)+\frac{\Delta T^{2} \Delta S}{4 \Delta W}+\frac{\Delta W}{4 \Delta S} .
$$

We shall adopt the convention $T_{\mathrm{B}}>T_{\mathrm{A}}$. Since the limiting values of $x$ are 0 and 1, we have for $x \geq 0$ (the solution with negative sign in (17))

$$
\Delta W \leq-\Delta T \Delta S_{\mathrm{B}}
$$

and for $x \leq 1$ (the solution with positive sign in (17))

$$
\Delta W \geq+\Delta T \Delta S_{\mathrm{A}}
$$

Clearly, the iso- $G$ curve exhibits monotonic variation with $x$ in the range $0 \leq x \leq 1$ for the case $-\Delta T \Delta S_{\mathrm{B}} \leq$ $\Delta W \leq+\Delta T \Delta S_{\mathrm{A}}$. For this case, the mathematical extremum of the iso- $G$ curve lies outside this composition range. In particular, for $\Delta W=0$, the iso- $G$ curve becomes a straight line.

The nature of the extremum can be found from the second derivative of $T_{0}$ evaluated at $x=x^{m}$. Differentiating $\left(\mathrm{d} T_{0} / \mathrm{d} x\right)$ with respect to $x$ and simplifying, we obtain

$$
\left.\frac{\mathrm{d}^{2} T_{0}}{\mathrm{~d} x^{2}}\right|_{x=x^{m}}=-\frac{2 \Delta S_{\mathrm{A}} \Delta S_{\mathrm{B}}\left[\Delta W+\Delta T\left(\Delta S_{\mathrm{B}}-\Delta S_{\mathrm{A}}\right)\right]}{\left[(1-x) \Delta S_{\mathrm{A}}+x \Delta S_{\mathrm{B}}\right]^{3}} .
$$

Since the denominator is necessarily positive and the second term in the numerator is usually much smaller than $\Delta W$ in magnitude, the extremum in $T_{0}$ is a minimum or maximum according as $\Delta W<0$ or $\Delta W>0$, respectively. Thus, conditions (20) and (21) apply respectively to occurrence of minima and maxima in the iso- $G$ curve. Alternatively, these conditions for extrema have been obtained earlier by van Laar (1908) in terms of the initial slopes of the liquidus and solidus, and by DeHoff (1993) in terms of the initial slopes of the iso- $G$ curve.

The locus of the congruent extremum can be found from (12) and (17) by continuously varying $\Delta W$. A minimum is obtained for $-\left(\sqrt{\Delta H_{\mathrm{B}}}+\sqrt{\Delta H_{\mathrm{A}}}\right)^{2}<\Delta W$ $<-\Delta T \quad \Delta S_{\mathrm{B}}$. The lower limit corresponds to $T_{0} \rightarrow 0$, $x^{0} \rightarrow \sqrt{\Delta H_{\mathrm{A}}} /\left(\sqrt{\Delta H_{\mathrm{B}}}+\sqrt{\Delta H_{\mathrm{A}}}\right)$ while the upper limit corresponds to $T_{0}=T_{\mathrm{A}}, x=0$. However, as discussed later the physically observable domain of a congruent minimum is restricted due to the occurrence of an ordered phase or a eutectic reaction. The boundary thus obtained is denoted by 1 in figure 1 . Similarly, a maximum is obtained for $\Delta T \Delta S_{\mathrm{A}}<\Delta W<+\infty$, for which the lower limit corresponds to $T_{0}=T_{\mathrm{B}}, x=1$ and the upper limit to $T_{0} \rightarrow+\infty, x^{\infty} \rightarrow \sqrt{\Delta S_{\mathrm{A}}} /\left(\sqrt{\Delta S_{\mathrm{A}}}+\sqrt{\Delta S_{\mathrm{B}}}\right)$. Similar to the case of a congruent minimum, the domain of occurrence of a congruent maximum is limited by the intervention of an ordered phase or a syntectic reaction. The calculated boundary is denoted as 2 in figure 1 . Thus, the congruent minimum can occur only within the composition range of 0 to $x^{0}$ (adjoining the component with lower transformation temperature) while the congruent maximum can occur only in the composition range of $x^{\infty}$ to 1 . Figure 1 shows the regions of occurrence of isomorphous phase diagrams having (i) no extremum (regions $\mathrm{Q}$ and $\mathrm{N}$ ), (ii) congruent minimum (regions $\mathrm{R}$ and $\mathrm{T}$ ) and (iii) congruent maximum (regions $\mathrm{K}$ and $\mathrm{L}$ ) in terms of the values of the regular solution parameters, $W^{\alpha}$ and $W^{\beta}$. In each of these cases, an ordered phase region or a miscibility gap occurs at low temperature according as $W^{\alpha}<0$ or $W^{\alpha}>0$, respectively. For this illustration, $T_{\mathrm{A}}, T_{\mathrm{B}}, \Delta S_{\mathrm{A}}$ and $\Delta S_{\mathrm{B}}$ have been chosen to be $T_{\mathrm{A}}=800 \mathrm{~K}$, $T_{\mathrm{B}}=1200 \mathrm{~K}, \Delta S_{\mathrm{A}}=\Delta S_{\mathrm{B}}=\Delta S=10 \mathrm{~J} \mathrm{~mol}^{-1} \mathrm{~K}^{-1}$. This value of $\Delta S$ is representative for the entropy of melting for metals.

\section{Peritectic or monotectic systems}

A peritectic reaction can occur in a phase diagram if there is (i) a miscibility gap in the solid phase and (ii) the maximum of the miscibility gap of the solid intersects the solidus. As discussed later, a eutectic reaction occurs instead of a peritectic reaction if the iso- $G$ curve displays a minimum lying inside the solid phase miscibility gap compositions. Following van Laar (1908), the limiting case thus corresponds to the touching of the maximum of

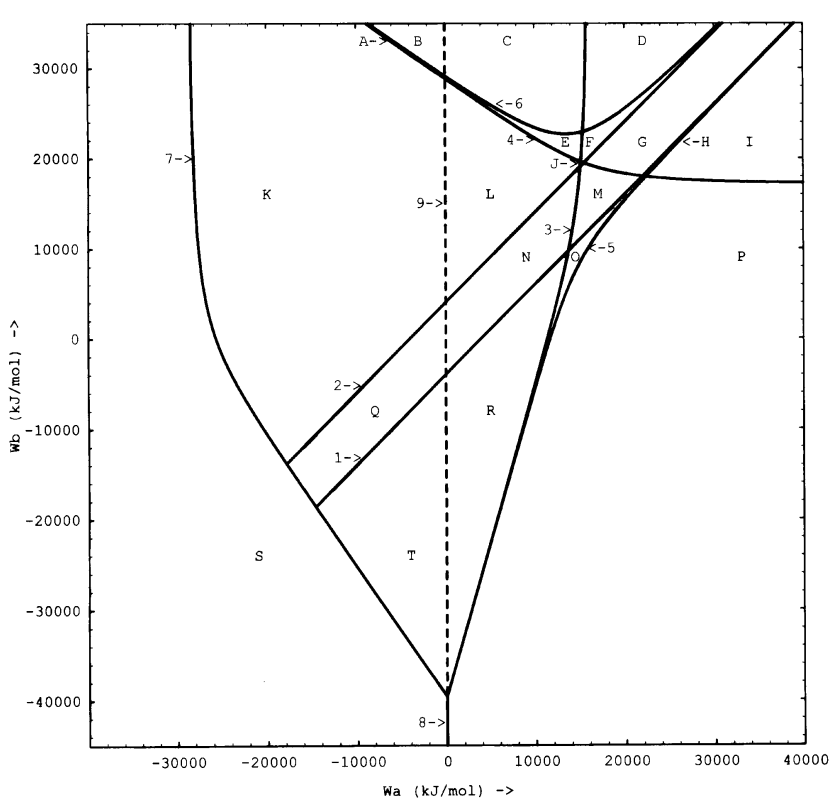

Figure 1. Existence domains for different topologies of binary phase diagrams in the space of regular solution parameters for the two phases $\left(\Delta S=10 \mathrm{~J} \mathrm{~mol}^{-1} \mathrm{~K}^{-1}\right)$. 
the solid phase miscibility gap and the solidus, which occurs at $x^{\alpha}=0 \cdot 5$, since the slope of the miscibility gap boundary as well as that of the solidus vanish simultaneously at this composition. Thus,

$$
T_{\mathrm{sol}}=T_{\mathrm{C}}^{\alpha}
$$

For this condition, at the point of touching $\left(x^{\alpha}=0 \cdot 5\right)$, the solidus in fact shows a horizontal inflection, for which,

$$
\left.\frac{d^{2} T}{\left(\mathrm{~d} x^{\alpha}\right)^{2}}\right|_{x^{\alpha}=0.5}=0 \text {. }
$$

Substituting from (4), (5) and (15) at $x^{\alpha}=0.5$ we have,

$$
\begin{aligned}
W^{\alpha} & =2 \frac{\Delta H_{\mathrm{A}}+\left(x^{\beta}\right)^{2} W^{\beta}}{\frac{\Delta S_{\mathrm{A}}}{\mathrm{R}}-\ln \left[2\left(1-x^{\beta}\right)\right]+\frac{1}{2}}, \\
W^{\alpha} & =2 \frac{\Delta H_{\mathrm{B}}+\left(1-x^{\beta}\right)^{2} W^{\beta}}{\frac{\Delta S_{\mathrm{B}}}{R}-\ln \left[2 x^{\beta}\right]+\frac{1}{2}} .
\end{aligned}
$$

Eliminating $W^{\alpha}$ from (25) and (26), we have

$$
\frac{\Delta H_{\mathrm{A}}+\left(x^{\beta}\right)^{2} W^{\beta}}{\Delta H_{\mathrm{B}}+\left(1-x^{\beta}\right)^{2} W^{\beta}}=\frac{\frac{\Delta S_{\mathrm{A}}}{R}-\ln \left[2\left(1-x^{\beta}\right)\right]+\frac{1}{2}}{\frac{\Delta S_{\mathrm{B}}}{R}-\ln \left[2 x^{\beta}\right]+\frac{1}{2}} .
$$

Since $T_{\mathrm{B}}>T_{\mathrm{A}}$, the composition of the liquidus corresponding to a horizontal inflection in the solidus at $x^{\alpha}=0.5$ will always be less than $0 \cdot 5$. Thus, $W^{\beta}$ has been evaluated for a series of values of $x^{\beta}$ varying from 0 to $0 \cdot 5$. Subsequently, $W^{\alpha}$ has been found from (26). Figure 1 shows the boundary (denoted by 3 ) thus obtained for the selected pure element properties as given above. Thus the peritectic reaction occurs in the regions $\mathrm{O}, \mathrm{M}, \mathrm{J}, \mathrm{H}, \mathrm{G}, \mathrm{F}$, and $\mathrm{D}$, all of which lie to the right of the calculated boundary. This boundary terminates at one end when $W^{\alpha}=0$, owing to the fact that the solid phase miscibility gap (which is a prerequisite for the peritectic reaction to take place) disappears for this value of $W^{\alpha}$. The coordinates of this terminal point in figure 1 are $\left(W^{\alpha}, W^{\beta}\right)=(0$, $-39595 \cdot 9$ ).

The occurrence of a monotectic reaction can be analysed in an analogous manner. Thus,

$$
\begin{aligned}
T_{\text {liq }} & =T_{\mathrm{C}}^{\beta}, \\
W^{\beta} & =2 \frac{\Delta H_{\mathrm{A}}-\left(x^{\alpha}\right)^{2} W^{\alpha}}{\frac{\Delta S_{\mathrm{A}}}{R}+\ln \left[2\left(1-x^{\alpha}\right)\right]-\frac{1}{2}}, \\
W^{\beta} & =2 \frac{\Delta H_{\mathrm{B}}-\left(1-x^{\alpha}\right)^{2} W^{\alpha}}{\frac{\Delta S_{\mathrm{B}}}{R}+\ln \left[2 x^{\alpha}\right]-\frac{1}{2}} .
\end{aligned}
$$

Eliminating $W^{\beta}$ from (29) and (30), we have

$$
\frac{\Delta H_{\mathrm{A}}-\left(x^{\alpha}\right)^{2} W^{\alpha}}{\Delta H_{\mathrm{B}}-\left(1-x^{\alpha}\right)^{2} W^{\alpha}}=\frac{\frac{\Delta S_{\mathrm{A}}}{R}+\ln \left[2\left(1-x^{\alpha}\right)\right]-\frac{1}{2}}{\frac{\Delta S_{\mathrm{B}}}{R}+\ln \left[2 x^{\alpha}\right]-\frac{1}{2}} .
$$

$W^{\alpha}$ has been evaluated for a series of $x^{\alpha}$ values from $0 \cdot 5$ to $1 \cdot 0$. Subsequently, $W^{\beta}$ has been found from (30). Figure 1 shows the boundary (denoted by 4) thus obtained. Thus, a monotectic reaction occurs in the regions A, E, F, G, H and I, all of which lie above the calculated boundary. As discussed below, a syntectic reaction occurs instead of a monotectic reaction if the iso- $G$ curve displays a maximum lying inside the liquid phase miscibility gap compositions.

\section{Eutectic or syntectic reactions}

A eutectic reaction can occur in a phase diagram if there is (i) a miscibility gap in the solid phase, (ii) a minimum in the iso- $G$ curve and (iii) the composition corresponding to the minimum lies within the composition range of the miscibility gap. The limiting case thus corresponds to the intersection of the miscibility gap and the iso- $G$ curve at the minimum in the latter (van Laar 1908). In case this minimum lies outside the miscibility gap, a peritectic instead of a eutectic reaction occurs as mentioned earlier. Thus,

$$
T_{0}^{m}=T_{\mathrm{mg}}^{\alpha} .
$$

Substituting from (14), (18) and (19) in the above, we have, for the special case of $\Delta S_{\mathrm{A}}=\Delta S_{\mathrm{B}}=\Delta S$,

$$
W^{\alpha}=-R \omega\left[\frac{T_{\mathrm{B}}+T_{\mathrm{A}}}{2}+\frac{\Delta T}{4}\left(\omega+\frac{1}{\omega}\right)\right] \ln \left(\frac{\omega-1}{\omega+1}\right),
$$

where $\omega=\Delta W /(\Delta T \Delta S)$. A more general relation valid for the case of $\Delta S_{\mathrm{B}} \neq \Delta S_{\mathrm{A}}$ can be found by substituting from (17) in (12) to find $T_{0}^{m}$ and in (14) to find $T_{\mathrm{mg}}^{\alpha}$. These can then be utilized in (32) to yield $W^{\alpha}$ in terms of $\Delta W$. $W^{\alpha}$ has been evaluated for a series of values of $\Delta W$. Subsequently, $W^{\beta}$ has been found from $W^{\beta}=W^{\alpha}+\Delta W$. The computed boundary denoted by 5 is displayed in figure 1 . Hence the eutectic reaction occurs in the regions $\mathrm{P}$ and $\mathrm{I}$ lying to the right of this boundary. As in the case of the peritectic boundary, a limiting value for $\Delta W$ can be found by observing that $W^{\alpha} \geq 0$. At $W^{\alpha}=0$,

$$
W^{\beta}=\Delta W=-\left(\sqrt{T_{\mathrm{B}}}+\sqrt{T_{\mathrm{A}}}\right)^{2} \Delta S .
$$

Thus, this eutectic boundary also terminates at $\left(W^{\alpha}\right.$, $\left.W^{\beta}\right)=(0,-39595 \cdot 9)$.

The occurrence of syntectic reaction can be analysed in an analogous manner. Thus,

$$
T_{0}^{m}=T_{\mathrm{mg}}^{\beta} .
$$


Substituting from an equation analogous to (14) for the $\beta$-phase and (18) and (19), we obtain, for the special case of $\Delta S_{\mathrm{A}}=\Delta S_{\mathrm{B}}=\Delta S$,

$$
W^{\beta}=-R \omega\left[\frac{T_{\mathrm{B}}+T_{\mathrm{A}}}{2}+\frac{\Delta T}{4}\left(\omega+\frac{1}{\omega}\right)\right] \ln \left(\frac{\omega-1}{\omega+1}\right) .
$$

As noted above, an equation for $W^{\beta}$ for the general case of $\Delta S_{\mathrm{B}} \neq \Delta S_{\mathrm{A}}$ can be found easily. The above expression has been utilized for evaluating $W^{\beta}$ for various values of $\Delta W$. Subsequently, $W^{\alpha}$ has been found from $W^{\alpha}=W^{\beta}-$ $\Delta W$. The boundary between the regions of occurrence of syntectic and monotectic reactions is denoted as 6 in figure 1. Thus, the syntectic reaction occurs in the regions $\mathrm{B}, \mathrm{C}$ and $\mathrm{D}$, which lie above this boundary.

\section{Order-disorder transformations}

For negative values of $W^{\alpha}$, ordering takes place in the solid phase. For large negative values of $W^{\alpha}$, there is a possibility that the order-disorder boundary intersects the $\alpha-\beta$ equilibrium boundaries, leading to the formation of complex phase diagrams exhibiting intermediate ordered phases. Thermodynamic models for ordering do not satisfactorily reproduce all the observed topographical details of real systems. Detailed analysis of such phase diagrams is therefore not very useful. Hence we shall approximately demarcate the region in which such complex phase diagrams will occur, without attempting to analyse them further. The simple Bragg-Williams model (Gordon 1968) of ordering for $b c c$ phases (B2) will be utilized for this purpose, since it is the analogue of the regular solution model for ordered structures. This leads to a second order transition, while a first order transition is observed for ordering in close packed structures. Thus, the boundary that will be obtained in this case will only be an approximate one for solution phases having crystal structures other than bcc. The order-disorder transformation temperature in this case is given by

$$
T_{\mathrm{tr}}=-\frac{2 x^{\alpha}\left(1-x^{\alpha}\right)}{R} W^{\alpha} .
$$

For this, the slope is given by

$$
\frac{\mathrm{d} T_{\mathrm{tr}}}{\mathrm{d} x^{\alpha}}=-\frac{2\left(1-2 x^{\alpha}\right)}{R} W^{\alpha} .
$$

The effect of ordering in the solid phase will be seen in the phase diagram when the order-disorder boundary touches the solidus. The conditions for these two curves to touch are

$$
T_{\mathrm{sol}}=T_{\mathrm{tr}}
$$

and

$$
\frac{\mathrm{d} T_{\mathrm{sol}}}{\mathrm{d} x^{\alpha}}=\frac{\mathrm{d} T_{\mathrm{tr}}}{\mathrm{d} x^{\alpha}} .
$$

Substituting from (37) in (8), we have

$$
\frac{d^{2} G^{\alpha}}{\mathrm{d} x^{\alpha^{2}}}=-4 W^{\alpha} .
$$

Substituting from (41) and (9) in (7), we have

$$
\begin{aligned}
\frac{\mathrm{d} T_{\text {sol }}}{\mathrm{d} x^{\alpha}}= & \frac{-4\left(x^{\beta}-x^{\alpha}\right) W^{\alpha}}{-x^{\beta} \Delta S_{\mathrm{B}}-\left(1-x^{\beta}\right) \Delta S_{\mathrm{A}}} \\
& +R\left[\left(1-x^{\beta}\right) \ln \left(\frac{1-x^{\beta}}{1-x^{\alpha}}\right)+x^{\beta} \ln \left(\frac{x^{\beta}}{x^{\alpha}}\right)\right]
\end{aligned}
$$

Substituting from (38) and (42) in (40), we obtain a transcendental equation with $x^{\alpha}$ and $x^{\beta}$ as the only unknowns. For the present convention, $T_{\mathrm{B}}>T_{\mathrm{A}}, 0<x^{\beta}<x^{\alpha}$ $<0 \cdot 5$, by selecting $x^{\beta}$, one can numerically solve for $x^{\alpha}$. These values can be substituted in (39) with appropriate substitutions from (4), (5) and (37) to yield two linear equations in $W^{\alpha}$ and $W^{\beta}$. By solving these equations we obtain the pairs of $W^{\alpha}$ and $W^{\beta}$, which when plotted yield the boundary denoted by 7 in figure 1 . The region lying to the left of this boundary corresponds to the occurrence of complex phase diagrams in which the solidus gets modified due to the presence of an ordered phase. This boundary terminates at one end corresponding to $W^{\alpha}=0$, since the ordered phase regions can form only for $W^{\alpha}<0$. The coordinates of the terminal point are $\left(W^{\alpha}, W^{\beta}\right)=(0$, $-39595.9)$. Note that the boundaries 3,5 and 7 are coterminus at this point.

For $W^{\beta}<-39595.9 \mathrm{~J} \mathrm{~mol}^{-1}$, positive values of $W^{\alpha}$ give rise to a eutectic while negative values of $W^{\alpha}$ give rise to complex phase diagrams with intermediate phases. Hence the boundary between these regions is defined by $W^{\alpha}=0$ and $W^{\beta}<-39595.9 \mathrm{~J} \mathrm{~mol}^{-1}$. This boundary is denoted as 8 in figure 1 . The region $\mathrm{S}$ lying to the left of boundaries 7 and 8 thus represents the occurrence of complex phase diagrams exhibiting intermediate ordered phases. For $W^{\alpha}=$ 0 and $W^{\beta}>-39595.9 \mathrm{~J} \mathrm{~mol}^{-1}$, the dashed line denoted as 9 in figure 1 corresponds to the occurrence of a low temperature miscibility gap to the right of the boundary and a low temperature ordered phase field to its left.

\section{Results and discussion}

In the preceding sections, we have given procedures for calculating the boundaries corresponding to the appearance/absence of a particular type of invariant reaction. For example, the region lying below the boundary 1 (b1) has a congruent minimum, while that above this boundary corresponds to the absence of a congruent minimum. Further, the region below $b 5$ corresponds to the occurrence of a eutectic reaction, while that above $b 5$ corresponds to its absence. As discussed earlier, the presence of a eutectic implies a metastable congruent minimum within the miscibility gap boundaries in the phase diagram. Thus, a stable congruent minimum will not be encoun- 
tered in the regions in which a eutectic reaction occurs. In addition, ordered intermediate phases can arise in the region lying to the left of $b 7$. Thus, a congruent minimum can occur only in the region bounded by $b 1, b 5$ and $b 7$.

In an analogous manner, it can be shown that a peritectic reaction occurs in the region bounded by $b 3$ and $b 5$. The intersection set of points of the above two regions (denoted by $\mathrm{O}$ in figure 1) corresponds to the occurrence of a peritectic reaction as well as a congruent minimum in the phase diagram. Similar arguments lead to an identification of the regions of occurrence of the different invariant reactions as well as their combinations shown in figure 1. These are listed below along with several of the known examples. Since the domains $\mathrm{J}$ and $\mathrm{H}$ are too small to be resolved on the scale used in figure 1, enlarged views of the same are presented respectively in figures 2 and 3 .

A Congruent maximum $(\mathrm{MX})+$ monotectic $(\mathrm{MO})+$ ordered phase region at low temperature (OR)

B Syntectic (SY) + OR

C $\quad \mathrm{SY}+$ miscibility gap at low temperature (MG)

D $\quad \mathrm{SY}+$ peritectic $(\mathrm{PE})$

E $\quad \mathrm{MX}+\mathrm{MO}+\mathrm{MG}$

$\mathrm{F} \quad \mathrm{MX}+\mathrm{MO}+\mathrm{PE}$

$\mathrm{G} \quad \mathrm{MO}+\mathrm{PE} \quad[\mathrm{Au}-\mathrm{Rh}]$

$\mathrm{H} \quad \mathrm{MO}+\mathrm{PE}+$ congruent minimum $(\mathrm{MN})$

$\mathrm{I} \quad \mathrm{MO}+$ eutectic (EU) $[\mathrm{Ag}-\mathrm{Ni}, \mathrm{Ag}-\mathrm{Rh}, \mathrm{Al}-\mathrm{Pb}$, $\mathrm{Cu}-\mathrm{Pb}, \mathrm{Ho}-\mathrm{V}, \mathrm{Li}-\mathrm{Na}, \mathrm{Ni}-\mathrm{Pb}]$

J $\quad \mathrm{MX}+\mathrm{PE}+\mathrm{MG}$

$\mathrm{K} \quad \mathrm{MX}+\mathrm{OR}$

$\mathrm{L} \quad \mathrm{MX}+\mathrm{MG}$

$\mathrm{M} \quad \mathrm{PE} \quad[\mathrm{Ag}-\mathrm{Pt}, \mathrm{Cu}-\mathrm{Ir}]$

$\mathrm{N}$ Simple isomorphous (SI) $+\mathrm{MG} \quad[\mathrm{Au}-\mathrm{Pt}, \mathrm{Cr}-\mathrm{W}$, $\mathrm{Cu}-\mathrm{Ni}, \mathrm{Cu}-\mathrm{Rh}, \mathrm{Ir}-\mathrm{Pd}$, Ir-Pt, Ir-Rh, Ni-Rh, Pd-Rh]

$\mathrm{O} \quad \mathrm{PE}+\mathrm{MN}$

$\mathrm{P} \quad \mathrm{EU}$ [Ag-Cu, Ag-Pb, Cd-Zn, Ho-Ta, Na-Rb]

$\mathrm{Q} \quad \mathrm{SI}+\mathrm{OR} \quad \mathrm{Au}-\mathrm{Pd}, \mathrm{Cd}-\mathrm{Mg}, \mathrm{Cu}-\mathrm{Pd}, \mathrm{Cu}-\mathrm{Pt}, \mathrm{Ta}-\mathrm{V}]$

$\mathrm{R} \quad \mathrm{MN}+\mathrm{MG} \quad[\mathrm{Au}-\mathrm{Ni}, \mathrm{Cr}-\mathrm{Mo}]$

$\mathrm{S} \quad$ Complex phase diagrams exhibiting intermediate ordered phases

$\mathrm{T} \quad \mathrm{MN}+\mathrm{OR} \quad[\mathrm{Au}-\mathrm{Cu}, \mathrm{Cs}-\mathrm{K}, \mathrm{Ni}-\mathrm{Pt}]$

$\mathrm{U} \quad \mathrm{MO}$ (see figure 4)

$\mathrm{Q}+\mathrm{N}$ SI [Ag-Au, Ag-Pd, Mo-Ta, Mo-V, Mo-W, $\mathrm{Nb}-\mathrm{Ta}, \mathrm{Nb}-\mathrm{W}$, Os-Re, Os-Ru, Pd-Pt, Pt-Rh, $\mathrm{Re}-\mathrm{Ru}, \mathrm{Ta}-\mathrm{W}, \mathrm{V}-\mathrm{W}]$

$\mathrm{R}+\mathrm{T} \quad \mathrm{MN} \quad[\mathrm{Cr}-\mathrm{V}, \mathrm{Cs}-\mathrm{Rb}, \mathrm{K}-\mathrm{Rb}, \mathrm{Nb}-\mathrm{V}, \mathrm{Ni}-\mathrm{Pd}]$.

As mentioned earlier, van Laar (1908) also calculated different types of phase diagrams by varying the values of $W^{\alpha}$ and $W^{\beta}$. However, his study was restricted to the positive octant of $W^{\alpha}$ and $W^{\beta}$ space $\left(W^{\alpha}>W^{\beta}>0\right)$. We have delineated the regions corresponding to all types of possible regular solution binary phase diagrams in the entire space of $\left(W^{\alpha}, W^{\beta}\right)$. It is interesting to note that all the real systems cited above lie essentially in the regions

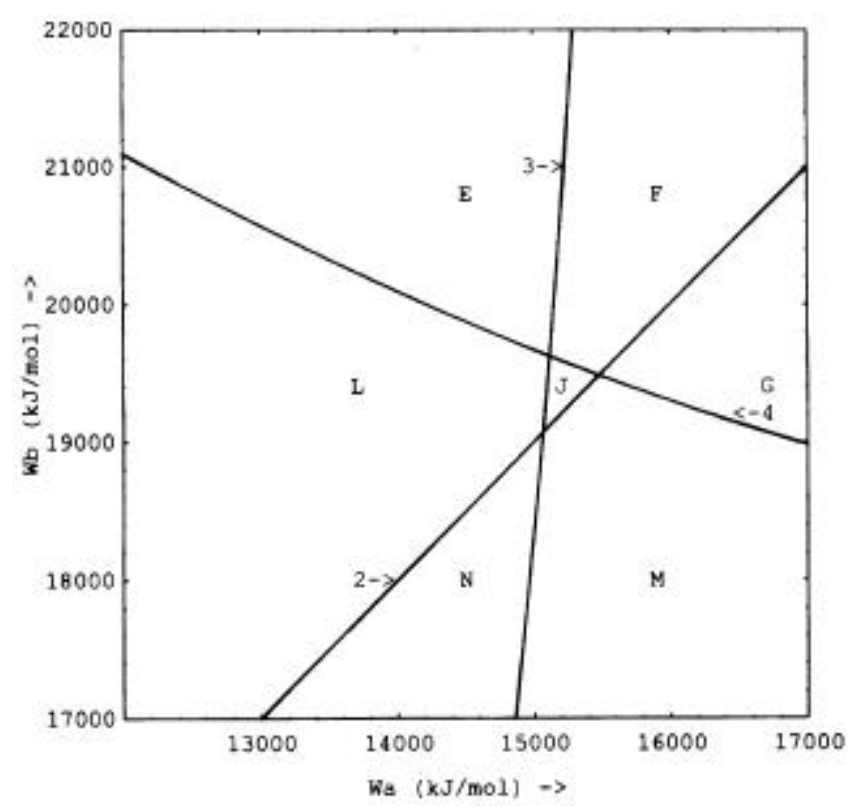

Figure 2. Enlarged view of the domain $\mathrm{J}$ in which a congruent maximum and a peritectic reaction occur $\left(\Delta S=10 \mathrm{~J} \mathrm{~mol}^{-1} \mathrm{~K}^{-1}\right)$.

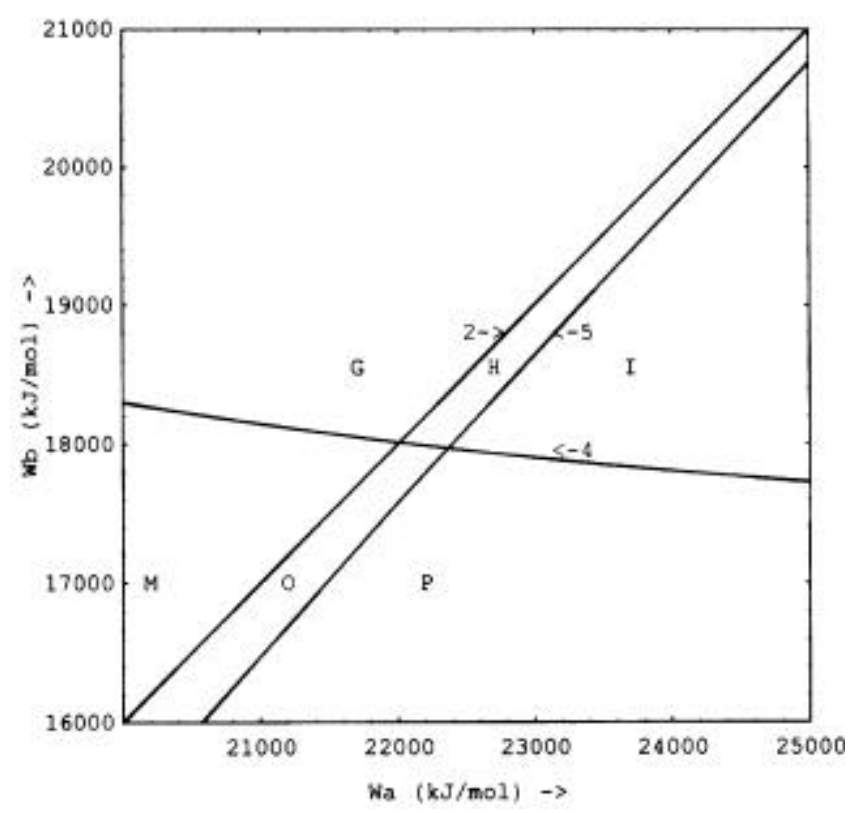

Figure 3. Enlarged view of the domain $\mathrm{H}$ in which a monotectic, a peritectic and a congruent minimum occur $(\Delta S=10$ $\mathrm{J} \mathrm{mol}^{-1} \mathrm{~K}^{-1}$ ).

corresponding to $W^{\alpha}>W^{\beta}$ but are not confined only to the positive octant.

All the combinations of $W^{\alpha}$ and $W^{\beta}$ considered by Pelton and Thompson (1975) and DeHoff (1993) for computing phase diagrams can be mapped on to figure 1 . The phase diagrams computed by them are in conformity with the respective domains of existence identified in our analysis, with the following exceptions. As can be seen 
from figure 1 , the phase diagram corresponding to the choice of $W^{\alpha}=20 \mathrm{~kJ} \mathrm{~mol}^{-1}$ and $W^{\beta}=15 \mathrm{~kJ} \mathrm{~mol}^{-1}$ is expected to exhibit a congruent maximum and a monotectic reaction. The phase diagram computed with the above choice of parameters indeed exhibits a congruent maximum at $T=1205 \mathrm{~K}$ and $x=0 \cdot 9$, and a monotectic invariant reaction at $T=1190 \mathrm{~K}$. This is in contradiction with the simple monotectic phase diagram reported by Pelton and Thompson (1975). Similarly, the phase diagram corresponding to $W^{\alpha}=10 \mathrm{~kJ} \mathrm{~mol}^{-1}$ and $W^{\beta}=15 \mathrm{~kJ} \mathrm{~mol}^{-1}$ exhibits a peritectic reaction at $T=799 \mathrm{~K}$ and a congruent minimum at $T=795 \mathrm{~K}$ and $x=0 \cdot 1$, contrary to the simple peritectic phase diagram reported by these investigators. Further, Pelton and Thompson (1975) have not considered the possibility of occurrence of complex phase diagrams with intermediate ordered phases arising due to an intersection of the solidus with the order-disorder boundaries. As a result, the phase diagram corresponding to $W^{\alpha}=-15 \mathrm{~kJ} \mathrm{~mol}^{-1}$ and $W^{\beta}=-20 \mathrm{~kJ} \mathrm{~mol}^{-1}$ is shown as a simple isomorphous diagram by them. However, the present analysis shows that the boundary of an ordered intermediate phase will intersect the solidus, thereby completely changing the topology of the phase diagram.

Chang and Chen (1997) have also calculated some of the possible phase diagrams corresponding to a choice of $\Delta S=20 \mathrm{~J} \mathrm{~mol}^{-1} \mathrm{~K}^{-1}$. Their results are in conformity with the domains for existence of a given type of phase diagram as calculated by us for the same choice of $\Delta S$. However, the $\left(W^{\alpha}, W^{\beta}\right)$ plot for $\Delta S=20 \mathrm{~J} \mathrm{~mol}^{-1} \mathrm{~K}^{-1}$ is not being presented here.

As can be seen from figure 2 , the small triangular region $\mathrm{J}$ corresponds to the occurrence of a congruent maximum and a peritectic reaction. It is interesting to study the effect of variation of $\Delta S$ on the presence of this region. By a systematic variation of $\Delta S$, it is found that the boundaries 2,3 , and 4 concur for $\Delta S \approx 11.5 \mathrm{~J} \mathrm{~mol}^{-1} \mathrm{~K}^{-1}$. Specifically for $\Delta S=13 \mathrm{~J} \mathrm{~mol}^{-1} \mathrm{~K}^{-1}$, the region $\mathrm{J}$ in figure 2 vanishes and a new region labeled $\mathrm{U}$ is formed, as shown in figure 4. This region $U$ corresponds to the occurrence of simple monotectic phase diagram.

It may be noted that the calculations presented above have all been carried out for the special case of $\Delta S_{\mathrm{A}}=$ $\Delta S_{\mathrm{B}}$. However, the analysis is valid for the general case as well and can be readily carried out for arbitrarily chosen values of entropies of transformation of the pure components.

\section{Conclusions}

Analytical conditions for the presence/absence of different invariant reactions in binary regular solution phase diagrams exhibiting two phases $\alpha$ and $\beta$ have been obtained. This is accomplished in terms of the appropriate intersections of the liquidus/solidus/iso- $G$ curves with the miscibility gap boundaries in liquid/solid phases. These

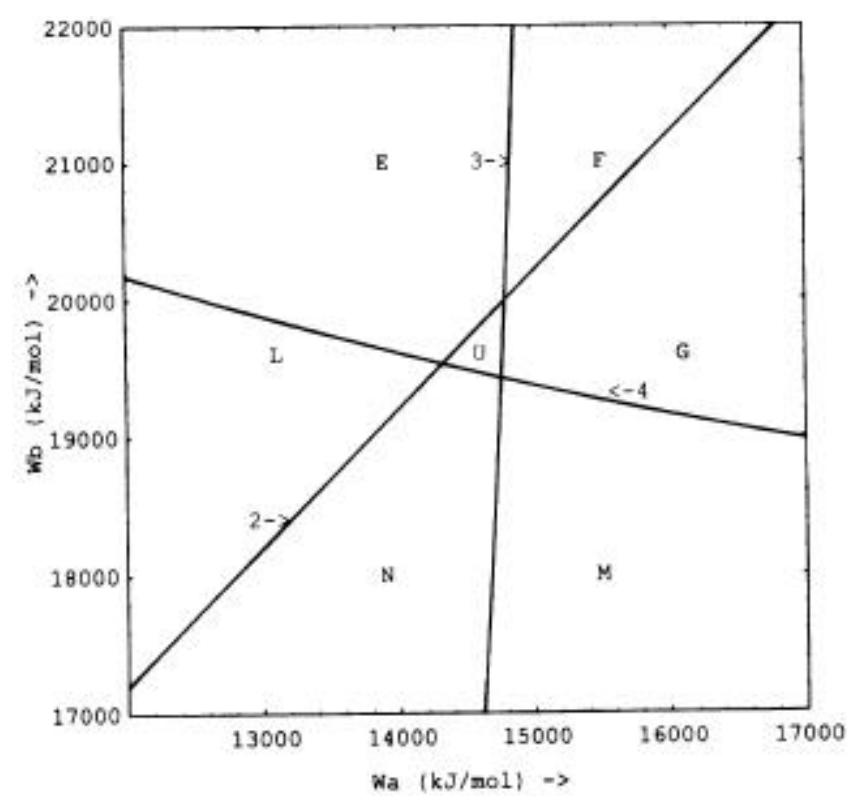

Figure 4. Enlarged view of the domain $U$ in which a simple monotectic reaction occurs, obtained for $\Delta S=13 \mathrm{~J} \mathrm{~mol}^{-1} \mathrm{~K}^{-1}$.

conditions represent curves in the space of regular solution parameters $\left(W^{\alpha}, W^{\beta}\right)$. Sets of these curves give rise to a domain of existence for a given type of invariant reaction. The intersection sets of such domains corresponding to two or more invariant reactions represent the domains in which a combination of these invariant reactions occur in the phase diagram. A procedure for a complete delineation of the existence domains of various invariant reactions or their combinations (except for the regions corresponding to the existence of ordered intermediate phases) has been formulated for the first time. This is illustrated for binary systems with chosen properties for pure components.

Computation of phase diagrams using solution models such as the CALPHAD/cluster variation method (Saunders and Miodownik 1998) proceeds by a nonlinear optimization of experimental data for the evaluation of the model (energy) parameters. If appropriate initial values of the parameters are not chosen, the topology of the phase diagram will be different from that of the observed one. Under such circumstances, the errors (the deviations between the calculated and observed compositions/temperatures, which are utilized in the optimization) cannot even be evaluated, leading to the breakdown of the optimization procedure. The analysis presented above serves as a very useful guide for selecting initial values of these energy parameters for ensuring a desired topology of the phase diagram of a chosen material system.

\section{Acknowledgements}

The authors wish to thank Dr V T Srikar, Massachussets Institute of Technology, USA and Dr Gouthama, Indian 
Institute of Technology, Kanpur, India, for readily providing copies of some of the publications cited in this communication.

\section{References}

Chang Y A and Chen S L 1997 Thermodynamics of metallic solutions, in Advanced physical chemistry for process metallurgy (eds) N Sano, W K Lu, P V Riboud and M Maeda (San Diego, USA: Academic Press) p. 5
DeHoff R T 1993 Thermodynamics in materials science (New York: McGraw-Hill) p. 277

Gordon P 1968 Principles of phase diagrams in materials systems (New York: McGraw-Hill) p. 124

van Laar J J 1908 Z. Phys. Chem. 63 216; 64257 (in German)

Oonk H A J 1981 Phase theory: The thermodynamics of heterogeneous equilibria (Amsterdam: Elsevier) p. 125

Pelton A D and Thompson W T 1975 Prog. Solid State Chem. 10119

Saunders N and Miodownik A P 1998 Calculation of phase diagrams: A comprehensive guide (CALPHAD) (Oxford: Pergamon) 